\title{
Diálogos para repensar la educación ambiental ${ }^{1}$
}

\author{
Fabiola Loaiza Robles ${ }^{2}$
}

\begin{abstract}
Resumen
El presente artículo aborda el tema de la didáctica no parametral como perspectiva de trabajo para la educación ambiental; la problemática que se presenta en este campo parte de la reducción del espacio didáctico de la enseñanza de lo ambiental a una simple descripción y transmisión de conocimientos, y teniendo en cuenta la complejidad del reto que nos presenta en esta temática, se hace necesaria su comprensión y acción desde opciones que se diferencien de lo tradicional y permitan otras miradas al problema; para tal fin, se utilizó como metodología el enfoque hermenéutico para abordar el tema y realizar el análisis interpretativo y la construcción de sentido desde los discursos de académicos expertos en esta nueva propuesta, encontrándose al final que ésta resulta ser de gran aporte para la enseñanza de lo ambiental ya que está sustentada en unas premisas y un enfoque epistemológico acorde para tal propósito.
\end{abstract}

Palabras clave: Didáctica no parametral, educación ambiental, pensamiento crítico, conciencia histórica, sujeto presente.

\section{Abstract \\ Dialogues for environmental education rethink}

The nearby paper addresses the subject of non parametric didactic as viewpoint of work for environmental education, piece of the difficulty that arises in this field by reducing space of didactic teaching environmental education to a uncomplicated explanation and transmission of awareness, and making aware the complexity of the challenge that presents to us with this topic, it is necessary its understanding and action from options that differ to the traditional way and allow others views of the problem, for this purpose, was used the hermeneutical methodology as approach to the theme and make the analysis interpretative and the construction of meaning from the discourses of academic experts in this new proposal, found at the end that it turns out to be of vast contribution to environmental education because it is based on some assumptions and an epistemological approach in line for that purpose. Keywords: Didactic non parametric, environmental education, critical thinking, historical consciousness, present subject.

1 Recibido: 22 de agosto de 2015. Aceptado: 15 de diciembre de 2015.

2 Fabiola Loaiza Robles. Estudiante del doctorado en Conocimiento y Cultura en América Latina del Instituto Pensamiento y Cultura en América Latina IPECAL, Magíster en Educación y Desarrollo Humano del convenio CINDE - Universidad de Manizales, Contadora Pública de la Universidad de Manizales. Docente de Tiempo Completo en La Universidad de La Salle, integrante del grupo de investigación GAO y del equipo de investigación de la Coordinación de Pedagogía y Didáctica de la Universidad de la Salle, correo electrónico: floaiza@unisalle.edu.co 


\section{Ad Portas}

"Diálogos para repensar la educación ambiental" es una investigación que nace como muchas, en la preocupación por la forma como se está abordando la temática ambiental, no es secreto que los problemas ambientales han hecho que la humanidad vuelque su mirada hacia este tema, que prácticamente se ha convertido en una moda al punto que muchas marcas comerciales lo han utilizado como estrategia para capturar la atención y así aumentar sus ventas creando un imaginario de conciencia y responsabilidad con el planeta.

Pero justo en este momento el medio ambiente nos da señales para indicarnos que de la forma como hemos venido estudiando, explicando y comprendiendo el mundo en un afán por controlarlo, no es el más indicado, que mientras en nuestras cabezas fragmentamos la realidad, la problemática ambiental nos indica que así no resolvemos el problema, pues se necesita un pensamiento y un sentimiento que sea crítico, complejo y sistémico porque el medio ambiente es así, presenta en un mismo instante la fusión de pasado y futuro con facetas que desde nuestra forma de entender son diferentes, pero en el fondo no lo son.

El medio ambiente debe ser pensado de forma permanente desde el campo educativo, en especial el didáctico, como espacio que, -en este caso-, se ocupa de enseñar a pensar en relación con el ambiente, como sujetos integrantes de los factores físico-naturales y simbólicosociales que lo conforman.

La educación ambiental como tal ha recibido muchas críticas y en Latinoamérica estas se han caracterizado por cuestionar los modelos de crecimiento y de consumo de los países industrializados, por tal razón se ha avanzado en otro concepto de desarrollo desde una perspectiva transformadora y critica.
Se resalta en este trabajo que la didáctica no parametral -como teoría y métodopor la cual se adquiere intencionalidad educativa de organización dentro del sistema y que se encuentra ligada a la pedagogía de la potencia, asume la práctica de la formación de sujetos como una práctica social, como parte de la cultura, que tiene una apuesta por la plasticidad, por la flexibilidad, por una opción de educar en lo ambiental en otras dinámicas, se puede tomar como un referente interesante para abordar la enseñanza de lo ambiental.

La didáctica no parametral tiene un gran sustento en la pedagogía critica, tendencia que identifica a Latinoamérica y que tuvo sus inicios en la década de los setenta con Paulo Freire, quien sostenía que cualquier educación, es en sí misma política.

La pedagogía crítica es una propuesta de enseñanza que intenta ayudar a los estudiantes a cuestionar y desafiar la dominación, y las creencias y prácticas que la generan. En otras palabras, es una teoría y práctica (praxis) en la que los estudiantes alcanzan una conciencia crítica, pero esto solo es posible si los docentes se encuentran formados en esta línea, ya que aquí no se concibe, como en otras perspectivas, que el docente es solo el asesor o el orientador, en este caso el docente tiene un nivel de responsabilidad muy alto y sabe que es parte de un proceso de formación y construcción de subjetividades e imágenes de mundo.

En esta tradición, el maestro trabaja para guiar a los estudiantes a cuestionar las teorías y las prácticas consideradas como represivas (incluyendo aquellas que se dan en la propia escuela), animando a generar respuestas liberadoras tanto a nivel individual como colectivo, las cuales ocasionen cambios en sus actuales condiciones de vida.

Se puede inferir en esta propuesta de la pedagogía crítica que la práctica educativa, está determinada por procesos que son dialógica y simultáneamente económicos, políticos e ideológicos. 
Es preciso decir que "el hacer didáctico, como acción intencional de promover la construcción de sentidos y significados subjetivos e intersubjetivos -es decir sociales- que den cuenta, en la acción reflexiva, de la realidad que se vive como presente coyuntural, histórico y potencial" (Quintar, 2005), ha de generar movimientos que deben preverse desde los ambientes académicos, máxime si se trata de un tema que requiere además de reflexiones, la puesta en marcha de diferentes acciones.

Es de tener en cuenta que asistimos a una época caracterizada por el pesimismo intelectual, donde se escribe desde el pánico y la desolación, donde el neoliberalismo económico permea las diferentes esferas del ser humano, donde no se está a favor de las ideas sino de la lógica del capital, se minimiza al sujeto y sus oportunidades se reducen a la mínima expresión; éstos síntomas permean los intentos de formación en el campo educativo, entonces, qué podríamos esperar de la formación ambiental?

Al respecto se observa una reducción en el campo didáctico de la educación ambiental a una visión instrumental de transmisión de conocimiento, que amerita una propuesta, una mirada desde otra perspectiva, como es -por ejemplo- la didáctica no parametral, pues se considera necesaria la apuesta por la formación de un sujeto con pensamiento crítico que piense su contexto latinoamericano.

El hacer didáctico al configurarse como acción que promueve la construcción de sentidos y significados, se convierte en referente obligado a la hora de hablar sobre la enseñanza de lo medioambiental y todas sus implicaciones en la construcción de lo natural-social, ya que es allí donde debemos analizar cómo estamos pensando el medio ambiente y al ser humano en su relación con éste.

El presente artículo interpreta las perspectivas de la didáctica no parametral sobre la educación ambiental. Cuando se habla de perspectiva se está incursionando en un tiempo diverso al de hoy, nos dice Heidegger (2000, p. 460). que "la praxis como ejercicio de la vida se mantiene en miradas que atraviesan: en perspectivas. El horizonte está siempre dentro de una perspectiva, de un mirar a través, en dirección de algo posible que se eleva y sólo puede elevarse desde lo que deviene, es decir desde el caos. La perspectiva es una trayectoria de la mirada previamente abierta sobre la que se forma en cada caso un horizonte" Así las cosas esta investigación viajó en perspectiva, en la búsqueda de un tiempo que no es el de hoy, que no fue ni será el pasado, quedándole como opción abrir camino en futuro desde el presente potencial del cual hablaron los académicos expertos en didáctica no parametral, para dar paso a una articulación de sentido con la educación ambiental.

El horizonte teórico de esta artículo está conformado por algunos planteamientos de la educación ambiental y de la didáctica no parametral, los cuales son necesarios para realizar el análisis de información y la dilucidación de la discusión académica que se presenta al final y que se refiere a la construcción de sentido de la didáctica no parametral para la educación ambiental, como categoría articuladora, ya que en el reconocimiento y la re-de-construcción de sentido, el protagonista es el sujeto con su sentir, con su carga afectiva y emotiva, que en relación con su realidad lo moviliza en la enseñanza-aprendizaje y en la construcción de conocimiento.

El método utilizado para desarrollar la investigación es el hermenéutico ya que lo que se busca es la interpretación, comprensión y construcción de sentido de la didáctica no parametral frente a la educación ambiental; para lo cual el trabajo se presenta de forma cíclica en los tres momentos que Gadamer (1984) propone en su círculo hermenéutico: entender el significado textual, interpretar el significado intertextual y finalmente comprender y dar sentido contextual. El diseño de la investigación es narrativo 
de tópicos, ya que está centrado en las perspectivas de didáctica no parametral de algunos teóricos e investigadores en didáctica no parametral y su relación con categorías de conocimiento como la ética, la política, la estética, la ciencia, la tecnología, el desarrollo, la economía, la sociedad y la cultura.

No es sencillo integrar los conceptos de teóricos como Hugo Zemelman con las exigencias prácticas que propone Estela Quintar, o las acciones de vida que sugiere Germán Guarín y las miradas pedagógicas de Alejandra Zemelman; pero sí hay una confluencia, una necesidad urgente de acción, donde la didactobiografía, los círculos de reflexión y sus resonancias entregan alternativas de humanidad para la educación ambiental.

\section{Justificación}

\begin{abstract}
La pedagogía se hace antropología. Esa conquista no se iguala al crecimiento espontáneo de los animales: se implica en la ambigüedad de la condición humana, se complica en las contradicciones de la aventura histórica, se explica, o mejor dicho, intenta explicarse en la continua recreación de un mundo que, al mismo tiempo, obstaculiza y provoca el esfuerzo de la superación liberadora de la conciencia humana.
\end{abstract} Paulo Freire

Las relaciones sociales presentes en la educación intentan producir un tipo de formación donde el sujeto construya autónomamente representaciones e imágenes del mundo, de sí mismo, e identificaciones de clase social, que son ingredientes para determinar su posición ocupacional; en este sentido la educación proporciona los recursos y medios para la producción de autoconceptos, identificaciones sociales y profesionales acordes con los requisitos de la división social del trabajo.

De tal forma, siendo conscientes de esta realidad, es necesario propender por un pensamiento crítico donde sea posible comenzar a hacer una historia distinta del presente, es decir, de aquellos discursos y prácticas que han dado forma a nuestro pensamiento frente a lo ambiental, para que aquello que aún no ha sido pensado o imaginado pueda llegar a existir, y el campo de la educación ambiental pueda ser repensado desde un horizonte crítico, apuntando a la transformación de los imaginarios que se han creado desde discursos que no corresponden con nuestras verdaderas necesidades.

El sistema educativo es, sin duda, uno de los medios más relevantes para que se pueda dar la formación ambiental, ya que lo que se espera es que en este proceso de enseñanza aprendizaje se genere la conciencia necesaria para fomentar el respeto a las diferentes formas de vida y por tanto a los sistemas sociales y naturales; por lo anterior, se hace necesario, entre muchos aspectos, revisar la didáctica con la cual se está enseñando lo ambiental, para de esta forma poder pensar en otra perspectiva de trabajo como lo es la didáctica no parametral.

La didáctica no parametral invita a reflexionar permanentemente en la acción educativa, a problematizar lo aparentemente normal, a mirar lo que no todo el mundo ve, y teorizar lo no pensado, por lo anterior, se considera interesante poder dar un vistazo a este método para pensar desde allí la didáctica de la educación ambiental.

Es de tener en cuenta también, que la realidad ambiental de Colombia no es mejor a la del continente o si se quiere del mundo, hay desaparición de especies, contaminación de ríos, insuficiencia no de normas sino de acciones por parte de las autoridades para controlar el tráfico de fauna y flora, la poca formación en los colegios y universidades sobre educación 
ambiental, la no búsqueda sistemática de energías alternas y los permisos para construcciones o ubicación de empresas sin reunir las mínimas normas ambientales, esta irresponsabilidad se debe en parte a la falta de formación frente a lo ambiental, una educación que propenda por la defensa y el respeto hacia el medio ambiente.

Es de resaltar que lo ambiental como temática y como problema de conocimiento y praxis necesita de unas miradas acordes con la complejidad que éste representa, muchas veces se ha planteado que la realidad es una sola y que no es posible su comprensión sin análisis holísticos, pero en los sistemas educativos el conocimiento se especializa cada vez más, lo que hace que estas miradas sean miopes; hoy con el reto que presenta la problemática ambiental, se hace necesario que éste sea pensado desde la inter y transdisciplinariedad, teniendo en cuenta que esta es una de las premisas que se proponen en la didáctica no parametral resulta interesante poder realizar este análisis desde esta propuesta, donde se tienen en cuenta diferentes frentes de pensamiento y acción.

\section{Contornos del Problema}

\section{Todo sistema de educación es una forma política de mantener o de modificar la adecuación de los discursos, con los saberes y los poderes que implican. \\ Michel Foucault}

Esta investigación parte de reconocer que existe una problemática en la didáctica de la educación ambiental referida a una reducción de su campo matizada por perspectivas instrumentales; lo anterior se debe a que hasta el momento hay un predomino de la enseñanza de lo medioambiental como un saber compartimentado desde una visión que ha sido lineal y que por tanto no corresponde con la complejidad de lo ambiental. En especial el problema se centra en que la educación ambiental ha sido concebida como medio para el desarrollo sostenible ${ }^{3}$ y que ha sido un campo predominante a la hora de enseñar lo ambiental.

Mientras el desarrollo sostenible se encuentra parcelado en las dimensiones humana, económica, ambiental y tecnológica, y que además privilegia el crecimiento económico como garante del desarrollo, la educación ambiental propende por la comprensión del ser humano como sujeto integral del ambiente, al igual que todos los procesos sociales, que incluyen lo económico y lo tecnológico, resulta de vital importancia que los académicos realicen el llamado de atención para que estas visiones fragmentadas de la realidad sean superadas definitivamente. Así como lo plantea Boaventura de Sousa (1998, p. 47), se observa,

"una contradicción creciente entre el ecosistema del planeta Tierra, que es finito, y la acumulación de capital, que tiende a ser infinita. Por otra parte la utopía ecológica, es utópica porque su realización presupone la transformación global, no solo de los modos de producción sino también del conocimiento científico, de los modos de vida, de las formas de

3 Es la educación, sin duda, el medio más relevante gracias al cual todo individuo en una sociedad puede acceder a los discursos, por lo cual afirma Foucault (1999, p. 45) que, "todo sistema de educación es una forma política de mantener o de modificar la adecuación de los discursos, con los saberes y los poderes que implican". La educación se ha convertido en el medio que asegura la distribución de los sujetos que hablan en diferentes tipos de discursos y también para la adecuación de discursos a cierto tipo de sujetos, de esta forma el sistema educativo se convierte en una ritualización del habla para los sujetos que se expresan desde los discursos, es decir, se da una adecuación del discurso con sus saberes y poderes; y como es sabido el desarrollo sostenible se concibe como un discurso que es preciso develar en el campo educativo, más que simplemente transmitirlo como verdad revelada. 
sociabilidad y de los universos simbólicos y, presupone, sobre todo, una nueva relación paradigmática con la naturaleza que sustituya a la relación paradigmática moderna".

Pero esta observación que hacen algunos pensadores muchas veces no es tenida en cuenta en el ámbito de la educación ambiental y se plantea que esta "no ha sido más que un campo específico de las ciencias de la educación que se dedica, en primera instancia, a una enseñanza y unas prácticas ecológicas y, en segunda instancia, a transformar la actitud del hombre frente a la naturaleza, conservando la escisión entre la naturaleza y la cultura" (Noguera, 2004, p. 74). No puede darse un pensamiento ambiental cimentado en la dicotomía sujeto-objeto ${ }^{4}$, donde el primero es dominante, mientras el segundo es el dominado y explotado, donde la sociedad está por encima de la naturaleza y, para el caso de la enseñanza del desarrollo sostenible la naturaleza se convierte en recurso o en capital, de tal forma que lo que se plantea es una sostenibilidad del capitalismo y no de la vida en sí misma.

"La problemática ambiental aparece como una crítica de una racionalidad económica prevaleciente y a los estilos de desarrollo dominantes; de esta forma implica el cuestionamiento del conocimiento que se ha venido produciendo y legitimando en este proceso histórico" (Leff, 1994, p. 14). En relación con lo anterior el desarrollo sostenible hace parte del imaginario cartesiano de la modernidad que pretende el dominio y explotación de los recursos, aunque poco a poco se ha ido demostrando su debili-

4 La separación entre sujeto-objeto ha estado presente en la historia del pensamiento científico y lo ambiental también se encuentra atravesado por esta premisa ya que se ha considerado por muchos años que el medio ambiente se refiere a la naturaleza o lo físico y lo social se ha descartado, aunque es de reconocer que en los últimos años la presencia de lo social se ha presentado con cierta fuerza en las discusiones e investigaciones de educación ambiental. dad y contradicción, ya que "el desarrollo tiene que ver con la tarea ilimitada de la razón tecnocientífica de desenvolverse a sí misma (ciencia y tecnología sin límites) y la sostenibilidad tiene que ver con los límites ecosociales, lo que hace que todo intento de desarrollo sostenible fracase porque el desarrollo predomina sobre la posibilidad de ser sostenible" (Noguera, 2004 , p. 78); el sujeto se ha mostrado débil ante el mundo, porque renunció a la naturaleza para poder someterla y explotarla a su antojo.

La didáctica en la educación ambiental se ha regido por lógicas de contenido, de objeto y de método 5 , por lógicas de enseñanza y de aprendizaje tradicionales. No existe una didáctica por lógicas de formación y conciencia histórica sobre lo ambiental, de ahí la novedad pedagógica, científica y formativa de la investigación.

Pensar en la amplitud del campo didáctico connota una recuperación de la voz del maestro desde su conciencia histórica, social y política, una recuperación de su carácter de sujeto de pensamiento crítico e innovador, por tal razón, es necesario analizar los esfuerzos que actualmente hacen algunos académicos de la educación desde una didáctica no parametral para dar una nueva comprensión de la relación del ser humano con su entorno físico-natural y simbólico-social que es precisamente a lo que hace referencia el medio ambiente que no compone solamente de la naturaleza sino que lo social también hace parte y se hace necesario trabajarlo en la educación ambiental.

5 La enseñanza de lo ambiental se ha discutido, pero no ampliamente y se ha llegado a concluir que uno de los grandes problemas tiene que ver con que se enseña como una materia mas donde se transmiten unos conocimiento sobre lo que es el medio ambiente y muchas veces se desconoce que éste conocimiento debe ser transversal en los curriculum de los diferentes programas para que así se pueda garantizar de alguna manera la formación ambiental, donde este haga parte del mundo de la vida de cada persona y no solamente en el mundo educativo. 
"El saber ambiental se va configurando desde su externalidad y negatividad, como un nuevo campo epistémico en el que se desarrollan las bases conceptuales y metodológicas para abordar un análisis integrado de la realidad compleja en la que se articulan procesos de diferentes órdenes de materialidad (físico, biológico, social). (Leff, 1994, p. 21); por esta razón se reconoce, entonces, la necesidad de trabajar la enseñanza y la toma de conciencia sobre lo ambiental desde una perspectiva de la didáctica no parametral, de tal manera que el cuestionamiento movilizador del problema propuesto para analizar es el siguiente interrogante: ¿Qué perspectivas de didáctica para la educación ambiental se pueden derivar del discurso de teóricos e investigadores en didáctica no parametral?, el cual se analiza desde las siguientes categorías: política, ética, científica, tecnológica, estética, económica y cultural.

Para dar respuesta a la anterior pregunta de investigación se planteó un objetivo general referido a: Interpretar en el discurso de teóricos e investigadores de la didáctica no parametral las posibilidades de repensar la enseñanza de la educación ambiental en los tiempos y espacios escolares y sociales. Para lo cual se plantearon como objetivos específicos: Reconocer elementos potenciadores de la didáctica no parametral en la enseñanza de la educación ambiental; Repensar la educación ambiental desde otra perspectiva didáctica diferente a las tradicionales; Identificar aspectos de la educación ambiental existentes, que se pueden resemantizar con la didáctica no parametral.

\section{Construcción conceptual}

Las desigualdades entre zonas, pueblos y personas, la tecnocracia gobernante, la simplicidad de pensamiento y la potencia consecuente de destrucción atentan contra la identidad terrenal. Por fortuna, cada nueva corriente de destrucción viene acompañada
de una contracorriente humana.

Morin, 2001.

El horizonte teórico de esta investigación tiene dos componentes que sirven de base para la concreción del objetivo propuesto. El primero está referido a la educación ambiental y el segundo a la didáctica no parametral en su perspectiva crítica.

Para empezar, la educación tiene como misión fundamental formar al individuo dentro de ciertos roles sociales; pero cuando se aborda lo ambiental, la responsabilidad parece ser más amplia, pero, a veces, menos practicada. Lo cierto es que el cuidado del medio ambiente es premisa fundamental, es decir, el papel de un buen acto educativo es formar o guiar al sujeto para una convivencia con y en el medio ambiente. Para lo anterior se recomienda que se apliquen criterios de interdisciplinariedad, interculturalidad y educación en valores, y una propuesta que integre al hombre con el ambiente y que a partir de allí se dé la construcción de conocimiento sobre lo ambiental, tal como lo plantea Maturana(1998, p. 221), "el conocimiento del mundo que vivimos es condición necesaria para el actuar responsablemente en él al participar en su construcción cotidiana", resulta pertinente entonces, que ese conocimiento sobre lo ambiental sea para generar conciencia y acciones que propendan por las soluciones a la problemática ambiental y no solo para llenar las bibliotecas de libros y tesis sobre medio ambiente.

Se pretende una educación ambiental que tenga como meta "formar una población mundial consciente y preocupada por el medio ambiente y con los problemas asociados, y que tenga conocimiento, aptitud, actitud, motivación y compromiso para trabajar individual y colectivamente en la búsqueda de soluciones a los problemas existentes y para prevenir nuevos"(Carta de Belgrado, 1975). Una población que 
sea consciente de que sus acciones tienen repercusión en el medio, que comprenda los problemas con un conocimiento básico y que tenga responsabilidad critica para resolver los problemas con aptitudes y actitudes. Se tiene entonces que la educación ambiental debe ser "una educación para el cambio de actitudes con respecto al entorno en el cual se desenvuelven los individuos y las colectividades, para la construcción de una escala de valores que incluya la tolerancia, el respeto por la diferencia, la convivencia pacífica y la participación, entre otros valores democráticos. Por consiguiente, implica una formación en la responsabilidad, íntimamente ligada a la ética ciudadana" (Política de educación ambiental nacional, 2002). El cambio de actitudes implica en primera instancia conciencia y ésta a su vez se debe dar con el conocimiento. Es por esto que resulta de gran importancia el tema de la educación ambiental pues es en este espacio donde se pueden dar visiones de mundo con sus problemáticas ambientales. Tal como lo planteara el maestro Zuleta (1995), "que la crisis de la educación es más aguda mientras más industrializada sea la sociedad, hay mayor deshumanización". Por lo tanto, es primordial conocer las formas de actuación, de movilización y de organización humana que promueve una política de educación ambiental.

"Lo ambiental constituye una de las grandes preocupaciones sociales, pero que tampoco se han resuelto de la mejor manera, como muchas de las vicisitudes humanas, aunque en este campo, no hay demasiadas alternativas, puesto que cada violación o alteración de lo biológico tendrá consecuencias sobre todas las especies e incluso sobre el universo cual sistema es" (González, 2009, p. 114) La temática ambiental se ha convertido en ese nuevo paradigma que como lo expresa Capra (2000, p. 28) "podría denominarse una visión holística del mundo, ya que lo ve como un todo integrado más que como una discontinua colección de partes". Si precisamente desde el pensamiento ambiental se plantea esta articulación, pues ésta debe ser tenida en cuenta en la educación ambiental, ya que es aquí donde se configuran las visiones de mundo de los estudiantes, de tal forma que para ellos la complejidad y sistematicidad de la realidad sea entendida como tal.

Con la educación ambiental se pretende entender el ambiente como el conjunto global de elementos y factores físico-naturales y simbólico-sociales que albergan la esencia de la actividad humana y todas las especies, y reconocer que el ambiente enmarca todo cuanto existe y determina las relaciones que en su interior se producen, como requisito para intentar cualquier comprensión de las leyes que mantienen el equilibrio de la naturaleza y el universo; "mientras que el viejo paradigma se basa en valores antropocéntricos (centrados en el hombre), la ecología profunda tiene sus bases en valores ecocéntricos (centrados en la tierra). Es una visión del mundo que reconoce el valor inherente de la vida no humana" (Capra, 2000, p. 32). Estos cambios deben ser tenidos en cuenta y asumidos en el espacio de la pedagogía y la didáctica ambiental, pues implican una transformación de fondo que no puede pasar desapercibida.

En el marco de la relación entre la educación ambiental y el desarrollo sostenible, este último se ha abordado con mayor fuerza desde 1987, a partir de la divulgación del informe Brundtland de la Comisión Mundial de Medio Ambiente y del Desarrollo, titulado "Nuestro Futuro Común", donde se entiende por desarrollo sostenible "satisfacer las necesidades del presente sin comprometer la capacidad de las futuras generaciones para satisfacer las propias" (Informe Brundtland, 1987). Frente al desarrollo sostenible existe un consenso mundial relacionado con los siguientes puntos: a) los problemas son globales y trascienden las fronteras nacionales; b) el ser humano debe ser destinatario del desarrollo; c) los problemas están interrelacionados y son interdependientes; 
d) el desarrollo económico no garantiza el humano; e) los obstáculos del desarrollo humano y medioambiental no radican en la escasez de recursos, sino en la voluntad política de los países y del sistema internacional; f) la solidaridad y la cooperación internacionales son indispensables para promover el desarrollo humano y proteger la naturaleza a escala mundial.

El desarrollo sostenible implica procesos de crecimiento global simultáneo en diversas dimensiones, como la económica, humana, ambiental y tecnológica. En estas dimensiones se debe dar el equilibrio en cuatro objetivos fundamentales, a saber: a) equidad social, b) crecimiento económico, c) ecoeficiencia, y d) responsabilidad institucional.

El informe replanteó la polarización entre crecimiento económico y medio ambiente, pues incluyó esta variable y el desarrollo social en el desarrollo sostenible, es decir, pasó de ser un asunto meramente ecológico a uno con dimensión económica y social; como bien lo plantea el profesor Quijano (2002, p. 99) "el desarrollo sostenible emerge postulando la biológico como hecho social significativo, en medio de la intensa problematización acerca de la continuidad de los paisajes biofísicos y culturales; es decir, situando en el debate las dificultades que enfrenta el mundo contemporáneo en términos de supervivencia global, proceso que deja ver múltiples producto de políticas y estrategias de desarrollo económico".

Alrededor del discurso del desarrollo sostenible se han generado otros que plantean análisis críticos como es el caso del discurso culturalista y el ecosocialista (Escobar, 1999); el primero hace énfasis en la cultura y pone en tela de juicio la de occidente, pues considera que en ella está el origen de la crisis ambiental actual. Esta perspectiva plantea la desaparición de la naturaleza como consecuencia inevitable del desarrollo de la sociedad industrializada, es decir, habla de una desaparición simbólica de la naturaleza.
El discurso ecosocialista hace énfasis en la capitalización de la naturaleza y divide el discurso en moderno y posmoderno. En el primero, la fuerza de trabajo y la naturaleza son condiciones de producción y también mercancías ficticias, y en la segunda, se considera la naturaleza ya no como una condición externa de explotación sino como fuente de valor en sí misma, es otra manera de capitalizar la naturaleza, mediada por el dinero y por el valor, opera a nivel de las representaciones y se constituye como conquista semiótica de la naturaleza. "Desde la perspectiva ecosocialista, para resumir, el discurso liberal del desarrollo sostenible no pretende la sustentabilidad de la naturaleza sino la del capital; desde la culturalista, lo que está en juego es la sustentabilidad de la cultura occidental" (Escobar, 1999, p. 89). Este tipo de análisis no pueden ser desconocidos por la educación ambiental y por esta razón se considera que la didáctica no parametral puede ser una muy buena opción para abordar estas temáticas, ya que esta propuesta busca entre sus propósitos develar los discursos de opresión que se encuentran ocultos en aquellos que trabajamos diariamente, muchas veces sin una verdadera comprensión de ellos.

Una didáctica no parametral para la educación ambiental parte de reconocer que el conocimiento y la enseñanza de lo ambiental debe poner al frente la relación del sujeto concreto con su existencia, es decir, con la vida, que no es exclusiva del ser humano. Se trata, entonces, en términos de una didáctica formativa o no parametral, de "generar conciencia en el darse cuenta y dar cuenta de las redes de relaciones de representaciones, símbolos y sentidos que están detrás de lo que se vive cotidianamente como natural y no como redes de significaciones y codificaciones históricamente construidas y, por lo tanto, históricamente posibles de ser deconstruidas y transformadoramente reconstruidas" (Quintar, 2005). Lo que conocemos como conocimiento ambiental 
es, sin duda, una construcción social que como tal tiene una carga simbólica, que es necesario que sea pensada desde los procesos didácticos de enseñanza y reconstruida en el aula.

La didáctica no parametral y la pedagogía de la potenciación ${ }^{6}$ se entrelazan y fundamentan desde la epistemología de la conciencia histórica, pensada y divulgada por el Dr. Hugo Zemelman Merino, donde lo epistemológico se aleja de la lógica del positivismo que ve a la epistemología como el campo de conocimiento encargada de estudiar la estructura y fundamentación de las ciencias, para centrarse en el proceso de complejización del conocimiento, donde el sujeto se resignifica en su ser y en su contexto en relación con la realidad y sus condiciones de existencia; la epistemología de la conciencia histórica implica un movimiento constante de los sucesivos actos de conciencia en niveles de abstracción cada vez mayores.

De otra parte, la mirada de la didáctica no parametral reconoce aquellos actos enseñantes que se salen de las canónicas, de lo impuesto, que como se sabe no producen hombres de pensamiento libres sino hombres de pensamiento constreñido o pensamiento apto para continuar con las lógicas en que nos hemos venido comprendiendo y devastando; la didáctica en esta perspectiva plantea que se debe "enseñar a los alumnos a enjuiciar moralmente los contenidos de aprendizaje -así como los procesos de comunicación sobre los que se establecen los procesos de enseñanza y aprendizaje- resulta fundamental para despertar en ellos una actitud afectiva de rechazo hacia las instituciones de injusticia, que es la base

6 Este término es acuñado por el Dr. Hugo Zemelman y consiste en resaltar que cada persona puede potenciar sus habilidades y conocimiento si tiene conciencia histórica, es decir, si se encuentra ubicado en su tiempo y espacio y se relaciona de forma tanto racional como emocional con el participando en su construcción y deconstrucción permanente. del pensamiento crítico-social" (Gimeno, 2009, p. 129).

La didáctica no parametral se planta sobre preguntas inquietantes, interrogantes que no siempre nos hacemos: "¿cuáles son las causas de que la racionalidad técnica perviva a pesar del reconocimiento de la necesidad de promover una racionalidad práctica y una acción emancipadora? ¿Por qué las teorías críticas se enseñan acríticamente y muchas veces dogmáticamente? ¿Qué, por qué y para qué enseñamos los enseñantes?" (Quintar, 2006, p. 21) En tales circunstancias abordar un pensamiento abierto exige una didáctica abierta, que no se ciña a parámetros, que se desparametralice para que el acto del enseñante, tan auténtico o tan demeritado, siga produciendo sujetos que potencien su pensamiento.

Los interrogantes entregados por la profesora Quintar se pueden trasladar a la educación ambiental, puesto que se lleva a las aulas bastante información ambiental, pero acríticamente o cuando se intenta, no siempre se logra, superar la opinión o la descripción de la realidad.

Por todo lo anterior es necesario entender la didáctica como una "disciplina estrechamente relacionada con los proyectos sociales y educativos, que tiene carácter significativamente político, comprometido con la formación de un modelo de hombre y la constitución de un tipo de sociedad" (Camilloni, 2007, p. 57). Es el campo encargado de estudiar los diversos componentes, humanos y materiales, que intervienen en el proceso de enseñanzaaprendizaje, con el fin de establecer sus funciones e interrelaciones, y dinamizar el acto docente-discente. En este orden de ideas se entrevé que la tarea de la didáctica es enorme, es decir, no puede quedarse en la pura definición, debe, pues, virar a la acción, en una necesidad de praxis, ya que, "asumir la didáctica desde un enfoque crítico significa posicionarse social, política, ética e históricamente en el mundo educativo, con disposición 
para reflexionar y problematizar el conocimiento, la enseñanza, el aprendizaje y la cultura escolar, tratando de empoderar y reconocer en los agentes del proceso educativo, sujetos capaces de construirse a sí mismos y de (de)construir su entorno social (Jiménez y Rendón, 2014).

\section{Método}

El análisis de la información se hizo bajo el método hermenéutico que busca interpretar en el discurso de teóricos e investigadores de la didáctica no parametral las posibilidades de repensar la enseñanza de la educación ambiental en los ambientes escolares y sociales. Implica una mirada desde la perspectiva epistemológica que brinda la hermenéutica ya que el trabajo tuvo su base en la interpretación de las entrevistas y de los textos publicados por los expertos que hicieron parte de la unidad de trabajo con el fin de dar sentido a la didáctica no parametral en el contexto de la educación ambiental.

La investigación tiene como fuente principal a los teóricos e investigadores de la didáctica no parametral, el Dr. Hugo Zemelman - Instituto de Pensamiento y Cultura en Latinoamérica - IPECAL México; Dra. Estela Quintar - Instituto de Pensamiento y Cultura en Latinoamérica - IPECAL - México; Dr. Germán Guarín - Universidad de Manizales - Colombia; y la Dra. Alejandra Zemelman - Fundación Tiempos Nuevos - Chile; de quienes se tuvo en cuenta la versión de sus entrevistas y de los textos publicados por ellos en estas temáticas, para realizar la interpretación de su discurso y así dar paso a una interpretación del sentido de la didáctica no parametral para la educación ambiental.

El diseño de investigación es narrativo de tópicos, ya que gira en torno a las perspectivas de didáctica no parametral de algunos teóricos e investigadores, donde a partir de sus experiencias en esta temática narraron la forma como la didác- tica no parametral da unas perspectivas de trabajo para la educación ambiental.

La técnica que se trabajó fue la entrevista focal, que consiste en la conversación, donde las personas que intervinieron (entrevistado y entrevistador) dialogan con arreglo a ciertos esquemas o pautas acerca de un problema o cuestión determinada, teniendo un propósito profesional. "Toda entrevista es un proceso dinámico multifuncional atravesado por el contexto social de una vida compleja y abierta continuamente a las transformaciones" (Sierra, 1998, p. 283).

La interpretación se centró en el relato de los expertos e investigadores en didáctica no parametral, viendo cómo estos sujetos dieron sentido a las temáticas mediante el relato de las mismas. La narrativa tiene un potencial para representar la experiencia vivida en la vida social educativa, se trata precisamente de reivindicar la dimensión de la experiencia personal; se trata de hacer emerger la materialidad dinámica de la palabra del sujeto como constituyente de su vivencia, memoria y olvido, ya que en el diseño narrativo se va cediendo la voz a los verdaderos protagonistas. La subjetividad es condición necesaria del conocimiento social y en el diálogo de subjetividades que se dan en la narrativa prima la construcción de comprensión de sentido. Para que se dé esta comprensión de sentido el trabajo tuvo una estructura polifónica ya que contó con la narrativa de varios sujetos.

La hermenéutica como es sabido incluye la interpretación y la comprensión de sentido, para lo cual partimos por afirmar que la comprensión en las ciencias sociales es histórica, un texto sólo se vislumbra cuando se percibe en su contexto; la lectura implica la participación de ese sentido pasado en uno presente, sin ser necesaria la congenialidad para reconocer lo verdaderamente significativo, es decir, el sentido originario de la tradición.

Se pretendió entonces, en este trabajo la comprensión de sentido de la didáctica 
formativa en la educación ambiental y para tal efecto se tuvieron en cuenta algunas condiciones como:

- Concebir el discurso desde la universalidad de su orientación, despojándolo del carácter momentáneo del acontecimiento, de la estrechez de los límites vividos por el autor, de su referencia ostensiva, y de los límites del cara a cara.

- La relación de la didáctica no parametral con un valor determinado y que pueda ser expresado en el lenguaje.

- La conciencia histórica, referida a la ubicación en el contexto del autor del texto o del sujeto con el cual se interactúa, para compartir y entender sus referentes simbólicos.

\section{Elucidando las discusiones}

\section{"La verdadera respuesta a la crisis ecológica sólo podrá hacerse a escala planetaria y a condición de que se realice una auténtica revolución política, social y cultural que reoriente los objetivos de la producción de los bienes materiales e inmateriales.}

Guattari (1990)

Para las sociedades actuales la educación sigue teniendo un elevado prestigio. Parece insólito que la educación siga teniendo tanto poder, pese a las voces que se levantan en su contra. En algunos círculos se dice que hoy se educa para someter, para que el Estado imponga sus lógicas; en otros ambientes menos pesimistas, se argumenta que se educa para liberar, para permitir que el sujeto supere los mitos religiosos o culturales; Ahora en los círculos más prudentes se indica que se educa para la superación, para la ascensión del individuo; en este mismo orden, podríamos seguir explorando conceptos para ampliar la mirada sobre la misión de la educación. Cabe, entonces, preguntarse ¿qué sucede con la educación ambiental? Sabemos de bastantes escrituras al respecto, algunos esgrimen fórmulas salvadoras, otros no tan seguidores de los mesianismos aconsejan modificar el currículum y hay quienes contundentemente apuestan que el problema radica en la didáctica.

Esta mirada de la didáctica de la educación ambiental desde la perspectiva de la didáctica no parametral no va contra nadie, ni acusa a nadie, es el nacimiento de unas opciones donde el sujeto esté presente y no ausente como en la mayoría de procesos académicos, políticos, económicos y culturales que hoy se proponen desde el poder.

El medio ambiente en el que vivimos nos presenta retos que modifican nuestras formas de vida y de comprensión, así acaeció en la historia y así viene siendo en lo que hoy se quiere denominar por posthistoria o si se profundiza, fin de la historia al estilo de Fukuyama. "La preocupación por lo ambiental debe estar en constante vibración en un trayectodeyecto-proyecto, donde el preguntar se deslice instigado por las prácticas filosóficas que demande una constante re-visión, en un evidenciar los usos tecnológicos y las re-percusiones ambientales de los dispositivos tecnológicos, rescatando que cualquier acción genera una reacción y en el plano ambiental no podemos darnos el lujo de continuar en la pedagogía ensayo-error sin prever las consecuencias" (González, 2009, p. 119), sabemos que el medio ambiente es todo y a veces pareciera ser nada, las multitudes hablan de él, pero en las acciones lo niegan. Si en el tránsito por la educación el medioambiente ha sido tema de preocupación ¿dónde queda el discurso?, ¿cuál es la tarea de la pedagogía y la didáctica?, sabiéndose que esta problemática exige soluciones globales, pero como tantas veces se ha dicho sin abandonar lo local.

Los retos estarían relacionados con la resolución de problemáticas medio ambientales y esta "cuestión ha plan- 
teado la necesidad de un pensamiento holístico y sistémico, capaz de percibir las interrelaciones entre los diferentes procesos que inciden y caracterizan a su campo problemático" (Leff, 1994, p. 24) y por esta razón se plantea una necesidad de conciencia en un respeto por el medio ambiente. De allí que diferentes instancias o campos de conocimiento han puesto su mirada en el abordaje del conocimiento de lo ambiental, pero no han logrado resolver las deficiencias. De igual forma Capra plantea que "cuanto más estudiamos los principales problemas de nuestro tiempo, más nos percatamos de que no pueden ser entendidos aisladamente. Se trata de problemas sistémicos, lo que significa que están interconectados y son interdependientes" (2000, p. 25), este es uno de los retos que tiene la educación ambiental, y es precisamente la comprensión y asimilación de la integralidad del medio ambiente.

A la educación como institución cultural se le entregó la responsabilidad de la enseñanza y el aprendizaje de lo que el hombre ha construido con sus formas de saber, como la filosofía, las artes, la ciencia y por supuesto lo medio ambiental, responsabilidad que se le impuso en las últimas décadas con el inconveniente de no ser un campo de conocimiento tradicional o que pueda ser abordado linealmente. La educación también ha tenido que replantearse diversos aspectos de sus lógicas de trabajo y contenido ya que la temática ambiental así lo amerita. Exige miradas holísticas, transdisciplinarias y de apertura, y en ese campo específico entra la didáctica a cumplir su misión que no puede ser delegada o transferida a otras disciplinas.

Siendo lo ambiental un aspecto fundamental-vital para la vida humana, se plantea que la educación desde los campos pedagógico y didáctico debe pensar y reconstruir permanentemente los aspectos relacionados con la enseñanza-aprendizaje del medio ambiente, ya que para el caso de la didáctica de la educación ambiental se observa que, en su mayoría, ésta se encuentra limitada por lógicas de contenido, aspecto que los docentes no siempre logramos desenmascarar y sin ser un propósito contribuimos con los modelos didácticos lineales a sólo transmitir contenidos, olvidándonos del sujeto, del ser afectado por sí, por el entorno y por los otros, en una alteridad revertida; ahí exactamente es donde la didáctica no parametral sienta sus bases, en el hombre que en su escisión no ha logrado concretar su proyecto de vida humano y social.

Adela Cortina (1999, p. 23) en su texto Ciudadanos del mundo, desde una mirada ética y con la intención de elaborar una teoría de la ciudadanía, deduce que desde la mirada por el otro, es necesario reconocer qué busca la universidad o qué pretende la universidad; piensa en una revolución cultural: "El sistema político y el económico están, pues, dependiendo de una revolución cultural, que asegure la civilidad, la disponibilidad de los ciudadanos a comprometerse en la cosa pública". Quizás en esa revolución que propone cabe la didáctica no parametral, en un camino a la civilidad, a la responsabilidad por lo público que en muchas ocasiones se quiere desprender del quehacer docente, y entonces el proceso de enseñanza de la didáctica sucumbe, porque las personas no parecieran ser las importantes sino los contenidos, ¿si la didáctica en educación ambiental es eficiente, por qué hay transgresión? Esta pregunta cabe para todas las disciplinas, lo que pasa es que en lo ambiental no se pueden dar tantas concesiones.

No sólo muere el medio ambiente, los animales y todas las formas de vida, también mueren las propuestas, sus lógicas las aprisionan, pudiera ser, suposición en verificación, que las didácticas tradicionales como compartimentación del saber están entrando en crisis y ellas, en sus ocupaciones no han buscado alternativas, o las que han encontrado salidas a la crisis de lo ambiental-humano, no hacen lo suficiente para que los docentes acudan a ellas en esa urgencia de reconstituir la 
educación ambiental y la forma como se comparten esos saberes, cuya práctica cotidiana no se compadece, primero con la teoría y segundo con la hecatombe que parece más cercana.

Es como si sus mismas propuestas perdieran credibilidad y entraran en deceso. Ahora no olvidemos que perder la credibilidad es uno de los riesgos que nadie, ni siquiera la educación, puede darse el lujo de hacer.

Ya va siendo tiempo de hacer una lista de las teorías que fenecieron y que algunas didácticas siguen conservando, como quien no sabe qué hacer con sus ruinas. En el mismo orden las didácticas requieren mirarse a sí mismas para conocer su estado y de lograr entrever las ruinas tienen dos opciones, la primera desaparecer, y la segunda, poco probable, la de revivir de sus propias cenizas como lo supo hacer el ave fénix.

\section{Elementos potenciadores de la didáctica no parametral para la educación ambiental}

La didáctica no parametral nace como una forma de leer la propuesta epistémica del presente potencial como discurso más amplio, más incluyente, planteada por el Dr. Hugo Zemelman, donde se da una relación de tránsito de un discurso epistémico a un discurso pedagógico. "La epistemología de la conciencia histórica o del presente potencial se entrelaza y fundamenta en un pensamiento pedagógico y didáctico que tiene como finalidad promover una educación emancipadora" (Quintar, 2005 , p. 2), toda propuesta teórica requiere en sus fundamentos de la fuerza que da una perspectiva epistemológica y esta es precisamente la unión que se da entre la didáctica no parametral y la epistemología de la conciencia histórica, articulación que puede resultar de gran interés para los procesos didácticos ambientales que requieren de un sujeto con potencialidad de enseñanza y aprendizaje de lo ambiental.

Esta propuesta epistémica incorpora la idea de movimiento "en articulaciones constantes y permanentes de sucesivos actos de conciencia que permiten complejizar el conocimiento en niveles de abstracción cada vez mayores" (Quintar, 2005, p. 9); tanto en la realidad, ya sea ésta económica, política, educativa, ecológica, etc., con distinto ritmo, necesita distintas coordenadas de tiempo y espacio, es decir, se trata de recuperar la exigencia de que las cosas están en constante movimiento, que la realidad nunca está estabilizada ni cristalizada.

"Las actuaciones decisivas en materia ecológica no sólo son de naturaleza ética, sino que también hay imbricaciones políticas, económicas, culturales, estéticas, científicas, metafóricas y míticas entre otras" (González, 2009, p. 118). No se puede agotar la conceptualización de la realidad socio-histórica, económica y cultural, como un conjunto de invariantes o como un conjunto de estructuras que están dadas desde ya y para siempre, y que se pueden captar de acuerdo con el principio clasificatorio de identidad y de determinación, sino que están en cambio y esos que están en cambio obligan a repensar esa realidad desde la exigencia de sus múltiples y complejas dinámicas.

La idea de movimiento rompe con estos límites; ya los fenómenos no son sólo económicos, políticos o culturales, son todos a la vez, entonces expresan la multiplicidad de dinamismo que es muy complejo porque son ritmos diferentes de la realidad, la realidad no es el compartimiento es una articulación, "se busca estar en la vastedad de la realidad y no dejarse aplastar por los límites de lo que ya está producido; de ahí que se tenga que romper con las determinaciones históricoculturales que nos conforman para rescatar el sujeto histórico como constructor y retador" (Zemelman, 2003, p. 13); en este campo la didáctica no parametral hace un 
proceso de fortalecimiento en esa mirada ambiental.

El movimiento de la realidad supone movimiento del sujeto y esto es lo que plantea el problema de los parámetros, es probable que esos parámetros no nos dejen ver una didáctica adecuada para enseñar lo ambiental de tal manera que lo teórico y lo práctico sigan escindidos, al cabo que el entorno se deteriora.

Como es sabido, en el currículum se organizan las maneras, los contenidos, donde en la mayoría de casos el sujeto queda afuera, porque lo que se muestra son realidades siempre externas al sujeto, entonces lo que se pretende es la inclusión del movimiento del sujeto, que no es más que la recuperación de su propia capacidad de pensar, de su propia capacidad de ver, no en términos de códigos disciplinarios ya preestablecidos sino de mundos abiertos, "podríamos pues, estar enfrentando un quiebre que obliga a estar abiertos como nunca, en vez de optar, como ocurre, por una supeditación a los parámetros del poder; o bien del proyecto hegemónico" (Zemelman, 2005, p. 14); aquí la didáctica no parametral entraría a fortalecer estas sendas en el sujeto, buscando que sea capaz de usar esos códigos disciplinarios a partir de su capacidad de preguntar, de su capacidad de conjeturar y de construir.

Es ahí donde el individuo tiene que colocarse frente al conocimiento, no sumergirse en el conocimiento, sino ser capaz de usarlo, pero para poder usar el conocimiento necesita algo fundamental, y es la capacidad de recuperar su propia capacidad de discernimiento, su propia capacidad de pensar, de darle sentido a la relación con el conocimiento a partir de algo que le es externo, que va a surgir de su capacidad de ubicarse en un momento, es un sujeto que ambientalmente hablando se compromete en teoría y en práctica; si ello no sucede, entonces, el proceso didáctico ha fallado; se requiere además, "colocarse en el momento, requiere que el sujeto construya su conocimiento desde las interrogantes que sepa formular desde el momento histórico en que está inserto" (Zemelman, 2005, p. 9), se hace necesario entonces, un sujeto que se entienda como parte del medio ambiente y que conozca sus temáticas y problemáticas para que esto le permita formular cuestionamientos que potencien su capacidad de pensar y proponer soluciones ambientales. El objetivo es entonces, hacer que los estudiantes aprendan a pensar su momento, un momento que no está cristalizado, pues el movimiento del presente es su potencialidad y también son los intereses sobre dinamismos.

Así las cosas, el hombre construye y la didáctica no parametral lo que le facilita es que el sujeto asuma la postura de constructo, esto supone desarrollar todas las fuerzas del sujeto, no solamente las del entendimiento, sino asumir una "postura epistemológica capaz de construir un conocimiento que devenga en la apropiación de lo necesario, de proyectarse en función de finalidades valóricas alternativas" (Zemelman, 2003, p. 12) donde incluya también su emocionalidad, su imaginación y la voluntad, es ahí donde se da la relación de la epistemología del presente potencial con la didáctica no parametral, el presente nunca está cerrado, siempre está abierto, que esté cerrado depende del sujeto, no hay ninguna ley ontológica que cierre el presente y mucho menos en la educación ambiental que ha reconocido la necesidad de actuar sobre un pasado que se escapa con mucha celeridad y del cual hoy podemos observar las consecuencias de sus errores por la falta de conciencia.

La didáctica no parametral "es una postura que comprende el proceso de enseñanza como un proceso intencional de permanente promoción de ruptura de sentidos y significados en el propio devenir existencial" (Quintar, 2006, p. 41) impulsada por la pensadora Estela Quintar y por el Instituto de Pensamiento y Cultura en América Latina que da unas miradas a conceptualizaciones como: La 
posibilidad de contextuar a los sujetos que aprenden, ya que siempre se parte de la propia experiencia del sujeto para realizar una construcción social del conocimiento a partir de allí y de una participación real desde las prácticas y no sólo desde construcciones idealizadas; la formación de un pensamiento libre; la promoción de un pensamiento policausal; la realidad como una totalidad contradictoria; la reflexión constante de lo que se enseña y se aprende; el conocimiento científico como medio e instrumento para decodificar la realidad; el planteamiento permanente de la duda; el reconocimiento de la educación como práctica social intencional; la creatividad como medio para resolver lo cotidiano; el mundo y lo que en él hay de enriquecedor; nuestras pobrezas lingüísticas para dar cuenta de la realidad; la potencia del lenguaje para convocar acontecimientos.

Lo anterior lo propone en su texto La enseñanza como puente a la vida (2006). Al adaptar estas premisas en la didáctica de la educación ambiental se puede concebir que en la posibilidad de contextuar a los sujetos que aprenden, este se refiere a que el conocimiento de lo ambiental no puede estar limitado a un simple campo de conocimiento ya que el paisaje deber convertirse en dispositivo que genere la potenciación de la conciencia ambiental.

La construcción social del conocimiento y la participación real conlleva a un sujeto dispuesto a generar, a recrear y a no siempre esperar, un sujeto que se sienta parte de interpretación y comprensión de lo ambiental y por lo tanto que puede convertirse en constructor de su conocimiento.

La formación de un pensamiento libre y la realidad como totalidad contradictoria se insertan en la enseñanza de lo medio ambiental en el sentido que esta realidad debe ser pensada como sistémica y compleja y la libertad del pensamiento no se refiere a que cada quien piense lo que quiera sino que se deben dar las diferentes posibilidades de análisis que permitan una reflexión constante del que enseña y aprende la temática ambiental.

El conocimiento científico hace parte de esta propuesta ya que la enseñanza no se puede basar solo en lo emocional o en la intuición; es necesario un contenido temático fuerte, pero para este caso, el de la educación ambiental, se hace pertinente un trabajo que sea en la medida de lo posible transdisciplinar, pues la problemática ambiental amerita miradas holísticas.

Leer el contexto educativo-ambiental significa no quedarse prisionero de parámetros que anticipan lo que el contexto es, que imponen significados a los términos, pero en función de un contexto que no corresponde con las verdaderas realidades, es decir, la educación ambiental se encuentra permeada por diferentes discursos que muchas veces no leen nuestro contexto sino que son apropiaciones de otras lecturas como es el caso del discurso del desarrollo sostenible el cual se considera en este trabajo como uno de los problemas de la educación ambiental, ya que ésta se ha centrado en muchos casos en la enseñanza que ofrece la mirada del informe Brundtland.

La educación ambiental debe propender por la configuración de un sujeto con identidad social hacia el medio ambiente y es precisamente desde la didáctica no parametral, en consonancia con la pedagogía de la potencia, donde se puede lograr este cometido, pues ésta última tiene como objetivo la recuperación del sujeto en su memoria, historia y olvido para trabajarlo en la configuración de dicha identidad.

La didáctica no parametral como crítica de la lógica de contenido y en su aspiración por recuperar la historicidad del sujeto, la conciencia histórica y la manera en que el sujeto se pone frente a las diferentes situaciones humanas, propone que, "es en la conciencia de la propia realidad en la que se pone de manifiesto el ser humano y su capacidad cognoscente, es en esa realidad en la que puede 
re-significar sentidos y significados de lo construido históricamente como conocimiento científico y tecnológico universal y no viceversa" (Quintar, 2005, p. 11). En esta perspectiva el sujeto involucrado en la educación ambiental debe estar en capacidad de poner en cuestión los contenidos de dicho campo, para analizar críticamente los conocimientos que hacen parte de su explicación y comprensión del mundo medioambiental.

\section{Posibilidades de repensar la educación ambiental en perspectiva de didáctica} no parametral

Pensar en la amplitud del campo didáctico de la educación ambiental connota una recuperación de la voz del maestro y del estudiante desde una conciencia histórica, social y política, que se unifique en lo ambiental, una recuperación de su carácter de sujeto de pensamiento crítico e innovador. "El sentido que la enseñanza adquiere en esta propuesta es el de promover el deseo de saber en la conciencia de sí y del mundo para actuar en él autónoma-instrumentada y transformativamente, es decir, aprehender en la conciencia de nuestra propia existencia material des-naturalizando lo dado como natural" (Quintar, 2006, p. 45), ese deseo por el saber que se debe alimentar de la conciencia que se adquiere en el proceso educativo ambiental es una de las claves potenciadoras que en últimas permitirá otro tipo de lecturas de la realidad y problemática ambiental.

Esta propuesta didáctica se centra en movilidades desde la palabra, la vivencia y la práctica para después acudir a la teoría, especificar la teoría como necesidad dentro del proceso y no iniciar en la teoría por imposición, por creerse que es la única manera de enseñar, así educar en lo ambiental es tratar de invertir el modo de enseñar, es partir un poco de la prác- tica y de las vivencias que han generado visiones previas de mundo, para luego comprender la teoría.

La educación ambiental tiene que pasar por articulación de sentido, evadiendo la escisión tradicional del pensamiento de occidente, para que articule el sujeto y el objeto, la teoría y la práctica, es decir, que la educación ambiental en perspectiva no parametral debe romper las antinomias y pasar a las articulaciones y construcciones que amerita el pensar ambiental.

Frente a las lógicas de poder de las políticas de educación ambiental y sus formas de actuación, se requiere revisar las lógicas de razonamiento incorporadas a esas decisiones políticas y preguntarse por el sentido lógico de una política de educación ambiental. También es necesario establecer qué tipo de sujeto y qué consideraciones de realidad están creando las políticas de educación ambiental, desde el punto de vista ideológico.

La didáctica no parametral tiene como una de sus tareas fundamentales romper con el sistema de creencias positivo que todo lo clasifica y ordena por materias, por parámetros, para poder mirar la educación ambiental en términos de articulación compleja, donde lo político es ambiental también, porque una decisión política puede afectar la tierra, por ejemplo, el manejo del petróleo, del gas, de la basura, pero no como problema de los ecologistas, sino como problema del hombre y su tiempo que emerge en determinadas prácticas.

El aporte de la didáctica no parametral a la educación ambiental está en su desparametralización, para que lo ambiental no sea una materia, una asignatura o un tema, sino que debe estar en todo, debe ser transversal, en palabras de la profesora Patricia Noguera (2004), se trata de ambientalizar la educación.

La generación de conciencia histórica que busca la didáctica no parametral se puede traducir en una educación ambiental donde la gente realmente sea capaz de asimilar el daño ambiental en su propia 
vida cotidiana y organizar sus actividades en función de ese riesgo del daño ambiental. Es preciso develar que detrás de la no conciencia histórica del daño ambiental están los intereses y si los intereses son discursos, son prácticas poderosísimas que le llegan a la gente todos los días a través de los medios de comunicación masiva, de la propaganda y de mil cosas. La función que cumple ahí la didáctica no parametral es incentivar a la persona a que vaya más allá del discurso, a que reconozca lo que está oculto, lo soterrado, es la incorporación del sujeto al discurso.

Lo que haría la didáctica no parametral en ese sentido simplemente es incorporar al sujeto, no imponerle un discurso ya armado, sino que éste sea capaz de construir el suyo, pero ¿qué significa discurso en este contexto?, significa reconocer posibilidades de realidad no unilateralmente, sino reconocerlas en toda la complejidad que tiene la realidad, que son sus múltiples dimensiones, las distintas opciones de construcción de realidad que existen.

Esto implica analizar con más detalle lo que significa leer el contexto, para ser capaz de resignificar los discursos, por eso es fundamental conocer el sentido y significado de las categorías centrales que estructuran algunos discursos, por ejemplo, ecología, equilibrio ecológico, daño ecológico, evidentemente tienen significaciones específicas, contextualizadas; es muy distinto el concepto huracán para una persona que nunca ha vivido los huracanes, es decir, el huracán es un parámetro, la diferencia está en cómo se vive, entonces, eso obliga a resignificarlo, lo mismo con pobreza, escolaridad, analfabetismo, marginalidad social.

Lo que articula todas estas situaciones es la historicidad. La historicidad del huracán, de la pobreza, de la deserción escolar, del analfabetismo, de la violencia, es una dimensión del contexto, pero no es un contexto aislado, ya que cada persona que interpreta tiene un mundo de significaciones demarcadas, precisamente por el medio ambiente en el que vive, por las articulaciones de vida.

\section{Conclusiones}

Resulta casi ilusorio pretender que este artículo ha llegado a unas conclusiones, ya que la didáctica no parametral entrega posibilidades de apertura para una educación ambiental, es una propuesta didáctica que se está gestando; por lo tanto, no se han medido los logros de lo teórico, de lo práctico, dicho por sus gestores, se deduce que hay un camino amplio en construcción, es una apuesta al optimismo sin ingenuidades, pero sustentada en teorías que, cualquiera sea la circunstancia, indaga por el sujeto; pretende entonces, visibilizar aquello que subyace en los discursos teóricos. Varias posibilidades para la educación ambiental se desprenden del grueso de esta investigación.

- Se observa, de acuerdo con las indagaciones bibliográficas y las entrevistas realizadas que la didáctica no parametral como teoría y método resulta ser una valiosa herramienta en la enseñanza de la educación ambiental, ya que presenta una perspectiva amplia de formación de sujetos con pensamiento crítico.

- La didáctica no parametral en la educación ambiental sería la encargada de la inclusión del movimiento del sujeto, que no es más que la recuperación de su propia capacidad de pensar, de su propia capacidad de ver, no en términos de códigos disciplinarios ya preestablecidos, sino que sea capaz de usar esos códigos disciplinarios.

- No es suficiente el exceso de teoría ambiental para resolver los problemas de esta índole en el aula de clase, sino que es necesaria la inclusión de las prácticas y experiencias locales, ya que los deterioros ambientales pueden variar de un lugar a otro en el mismo país, y esta razón nos lleva a concluir que la didáctica no parametral 
puede ser una herramienta importante en este proceso por la metodología que utiliza donde el sujeto está presente desde su didactobiografía.

- Latinoamérica tiene un interesante camino en educación ambiental, lleva cerca de cuatro décadas avanzando en investigaciones sobre el tema, donde ha pasado por diferentes estadios, cuando se empezaron a dar los primeros avances no se consideraba a "la sociedad desde sus preocupaciones de conservación, y tardaron años en insertar a los seres humanos, a sus saberes y a sus opciones de vida como temas de interés de sus acciones, además de considerarlos como simples depredares de la naturaleza" (Tréllez, 2006, p. 70); es de entenderse esta situación ya que hasta hace poco se consideraba que el medio ambiente era la naturaleza y no se tenía en cuenta la cultura como parte de lo ambiental.

- Al estar la didáctica no parametral sustentada en la epistemología del presente potencial y ésta en la conciencia histórica, en educación ambiental la conciencia estaría relacionada con poder asimilar el daño ambiental a la propia existencia y organizar la vida en función del riesgo del daño ambiental.

- Hay que reconocer las categorías de las tecnologías para leer las decisiones tecnológicas ¿Quién indaga por los diseños tecnológicos? Pregunta Zemelman. La respuesta no parece sencilla, puesto que desborda los intereses instrumentales.

- La didáctica no parametral, dentro de sus objetivos, pretende develar los discursos que se encuentran ocultos y configuran visiones de mundo en determinados contextos; para el caso que nos ocupa es importante ver cómo se encuentra que el discurso del desarrollo sostenible está determinado fundamentalmente por una perspectiva económica, dejando al margen importantes elementos culturales, sociales, entre otros, de verdadero desarrollo sustentable; por lo tanto la didáctica no parametral permite formas de análisis y de sentir que incorporan al sujeto para que sea capaz de construir discursos que contribuyan a una adecuada formación crítica del sujeto en lo ambiental, donde pueda reconocer posibilidades de realidad sistemáticas y complejas, aislándose de aquellas visiones unilaterales.

- Con respecto a la escisión sujeto-objeto que se presenta en las prácticas educativas que han separado al sujeto de la realidad ambiental, a través del presente trabajo es posible evidenciar que el entorno y el sujeto hacen parte de una unidad, donde hay múltiples interdependencias y relaciones, donde éstas pueden ser trabajadas desde el aula a partir de esta propuesta.

- Se reconoció que las dimensiones trabajadas desde la didáctica no parametral (política, cultural y económica) deben entenderse como articuladas en lo ambiental y que el sujeto como tal emerge de la dimensión ambiental, donde es condicionado en su subjetividad por el paisaje al cual pertenece y que a su vez realimenta lo político, en una crítica epistémica y didáctica, donde siempre se exigirá desde qué lugar epistémico se está colocando para pensar la enseñanza. De lo anterior se deduce que la didáctica no parametral está en construcción y por lo tanto sus ámbitos, límites y horizontes aún no están claros, por ello en la medida que se investiga se puede teorizar, es una propuesta que se está constituyendo.

- La didáctica no parametral puede advertir sobre los peligros de una ideología ambiental, en donde existe una lógica de discurso del poder con carácter antropocéntrico, pretendiendo solucionar los problemas con miradas antropológicas, queriendo resarcir lo que el hombre mismo dañó. 


\section{Plumilla Educatiual}

- A través de la didáctica no parametral se pueden revisar las lógicas de razonamiento, el sentido lógico y las consideraciones de realidad incorporadas en la enseñanza de la educación ambiental, indagar por el sentido ético y político que de la tierra se tiene y desplazar o desplazarse al interés por el acontecimiento básico o primordial que es la vida.

- Para finalizar, la didáctica no parametral puede coadyuvar en la comprensión de lo ambiental, permitir la incorporación del conocimiento al ámbito emocional, y generar un cambio en la cosmovisión tradicional, unilateral y limitada de lo medioambiental.
- La didáctica no parametral tiene dispositivos importantes para reflexionar y actuar dentro de la educación ambiental. No es suficiente con saber teorías, es necesario dar cuenta de ellas dentro del sujeto. Este es uno de los grandes aportes de la didáctica no parametral que, el sujeto conmocionado logre integrar su mundo teórico al práctico, es decir pasar de un conocimiento teórico a uno epistémico; permitir al sujeto un darse cuenta de la realidad para poder actuar o intervenir si es del caso, puesto que la problemática ambiental exige acciones políticas, éticas, estéticas, religiosas, económicas, científico-tecnológicas y, como no, educativas.

\section{Bibliografía}

\section{Fuentes}

Bauman, Zygmunt. (2002). Modernidad líquida. Buenos Aires: Fondo de Cultura Económica.

Bermúdez Guerrero, Olga María. (2003). Cultura y Ambiente. La educación ambiental contexto y perspectivas. Bogotá: Universidad Nacional - Instituto de estudios ambientales IDEA.

Camilloni, Alicia. (2007). El saber didáctico. Buenos Aires: Paidós.

Cortina Orts, Adela. (1999). Ciudadanos del mundo. Madrid: Alianza.

De Sousa Santos, Boaventura. (1998). De la mano de Alicia: lo social y lo político en la posmodernidad. Bogotá: Siglo del Hombre Editores, Facultad de Derecho Universidad de los Andes.

Escobar, Arturo. (1999). El final del salvaje. Bogotá: CEREC -ICAN.

Foucault, Michel. (1999). El orden del discurso (1ra Ed.). Barcelona: Tusquets Editores, S.A.

Freire, Paulo. (1970). Pedagogía del oprimido. México: Siglo veintiuno editores.

Fukuyama, Francis. (1992). El fin de la historia y el último hombre. Barcelona: Editorial Planeta

Gadamer. Hans-Georg. (1984). Verdad y método. Fundamentos de una hermenéutica filosófica. Salamanca (Esp.): Ediciones sígueme.

Gimeno Lorente, Paz. (2009). Didáctica crítica y comunicación. Barcelona: Octaedro

González González, Miguel Alberto. (2009). Horizontes Humanos: Límites y Paisajes. Manizales: Universidad de Manizales.

Guarín Jurado, Germán. (2005). Nuevas razones para la racionalidad en horizontes de complejidad. Manizales (Col.): Universidad de Manizales.

Guarín Jurado, Germán. (2007, julio) [Entrevista con Germán Guarín, docente Universidad de Manizales, Colombia, Hacia una didáctica formativa] grabación en audio.

Guattari, Félix. (1990). Las Tres Ecologías. Paris: Ediciones Galilée.

Heidegger. Martín (2000). Nietzsche I. BarceIona: Ediciones destino.

Jiménez, José Raul y Rendón, Margarita. (2014). Didáctica Universitaria. En: Londoño, Guillermo (Ed.), Docencia Universitaria. Sentidos, didácticas, sujetos y saberes. Bogotá: Ediciones Unisalle.

Leff, Enrique. (1994). Ciencias sociales y formación ambiental. Barcelona: Editorial Gedisa. 
Maturana Romesín, Humberto. (1998). El sentido de lo humano (1ra Ed.). Bogotá: Dolmen Editores.

Ministerio del Medio Ambiente y Ministerio de Educación Nacional (2002). Política de Educación Ambiental Nacional, Bogotá.

Morin, Edgar. (2001). Los siente saberes necesarios para la educación del futuro. Barcelona: Paidós Studio.

Noguera de Echeverry, Ana Patricia. (2004). El reencantamiento del mundo. Manizales: Universidad Nacional- IDEA. México: PNUMA.

Quintar, Estela. (2006). La enseñanza como puente a la vida (3ra Ed.). México: IPECAL, Instituto politécnico Nacional.

Quintar, Estela. (2007, septiembre) [Entrevista con Estela Quintar, secretaria académica IPECAL, México, En búsquedas: hacia una didáctica no parametral] grabación en audio.

Sierra Caballero, Francisco. (1998). Técnicas de investigación en sociedad, cultura y comunicación. Editorial Pearson.

Tréllez Solís, Eloísa. (2006). Algunos elementos del proceso de construcción de la educación ambiental en América Latina. Revista Iberoaméricana de Educación, 41, 69 - 81.

Zemelman Merino, Hugo. (1998). Sujeto: existencia y potencia. Barcelona: Antrhopos.

Zemelman Merino, Hugo. (2002). Necesidad de conciencia. Un modo de construir conocimiento. México: Antrhopos.

Zemelman Merino, Hugo. (2006). El conocimiento como desafío posible. México: IPECAL, Instituto Politécnico Nacional.

Zemelman Merino, Hugo. (2007, agosto) [Entrevista con Hugo Zemelman Merino, direc- tor de IPECAL, México, Epistemología de la conciencia histórica] grabación en audio.

Zemelman Schmauk, Alejandra. (2007, agosto) [Entrevista con Alejandra Zemelman, coordinadora programas de formación en la Fundación Tiempos Nuevos, Chile, Experiencia frente a la educación ambiental no parametralizada] grabación en audio.

Zuleta, Estanislao. (1995). Educación y Democracia. Un campo de combate. Bogotá: Editorial Corporación Tercer Milenio.

\section{Referencias}

Capra, Fritjof. (2000). La trama de la vida. Una nueva perspectiva de los sistemas vivos. (3ra ed.). Barcelona: Editorial Anagrama.

Carta de Belgrado (1975). Una estructura global para la educación ambiental.

Comisión Mundial del Medio Ambiente y del Desarrollo. (1988). Nuestro Futuro Común Bogotá: Alianza Editorial Colombiana, Colegio Verde de Villa de Leyva.

González González, Miguel Alberto. (2014). Miedos y olvidos pedagógicos. Rosario: Homosapiens.

Quijano Valencia, Olver Bolívar. (2002). De sueño a pesadilla colectiva. Elementos para una crítica político-cultural al desarrollo. Popayán (Col.): Editorial Universidad del Cauca,

Quintar, Estela. (2005). En diálogo epistémico didáctico. Consultado 09.09.2007, en línea: www.ipecal.net.com

Zemelman Merino, Hugo. (2003). Horizontes de la razón I. Barcelona: Antrhopos.

Zemelman Merino, Hugo. (2005). Pensar teórico, pensar epistémico. Consultado 10.09.2007, En línea: www.ipecal.net.com 